\title{
OUTPUT FEEDBACK CONTROL OF NONMINIMUM-PHASE NONLINEAR PROCESSES
}

\author{
COSTAS KRAVARIS \\ Department of Chemical Engineering, The University of Michigan, Ann Arbor, MI 48109, U.S.A \\ PRODROMOS DAOUTIDIS ${ }^{\dagger}$ \\ Department of Chemical Engineering and Materials Science, University of Minnesota, Minneapolis, \\ MN 55455, U.S.A. \\ and
}

RAYMOND A. WRIGHT

The Dow Chemical Company, 1400 Building, Midland, MI 48667, U.S.A.

(First received 31 July 1992; accepted in revised form 22 November 1993)

\begin{abstract}
This work concerns the development of a general framework for the analysis and output feedback control of open-loop stable nonminimum-phase nonlinear processes. A Smith-type abstract operator structure is introduced, allowing the reduction of the controller synthesis problem for nonminimum-phase processes to the one for minimum-phase processes. State-space methods are used to derive a reduced-order output feed back controller that induces a desired input/output behavior for processes with unstable inverse dynamics and deadtime. The underlying structure of the reduced-order controller is also identified and studied. Finally, an example from a class of chemieal reaction systems with nonminimumphase characteristics is used for evaluating the performance and robustness of the developed control method.
\end{abstract}

\section{INTRODUCTION}

The control community hus witnessed a flourishing research activity in the area of nonlinear systems theory and control. Following the input/output approach, abstract operator methods have bccn cmployed in the study of stability issues [e.g. Safonov (1980) and Desoer and Vidyasagar (1975)], coprime factorizations and $Q$-parametrization [e.g. Hammer (1984), Desoer and Lin (1984) and Desoer and Kabuli (1988)], etc. From a controller synthesis point of view, although the input/output perspective provides valuable insights and philosophical perspective, it lacks the transparency and explicitness that the transfer function approach has in a linear control setting. On the other hand, the introduction of differential geometric techniques in nonlinear analysis and control has allowed fundamental aspects of nonlinear dynamics to be understood and typical theoretical control problems to be successfully addressed in a state-space framework Lsee e.g. the books by Isidori (1989) and Nijmeijer and van der Schaft (1990)]. The strength of these methods clearly lies in the ability to employ nonlinear state feedback to modify the dynamic behavior of nonlinear systems. The synthesis of output feedback controllers is then addressed through combination of state feedback laws and state observers. Although a limited number of general results are available for the design of nonlinear state ob-

\footnotetext{
'Author to whom correspondence should be addressed.
}

servers [see e.g. Misawa and Hedrick (1989)], the use of the process modes for the state observation has been shown to lead to a comprehensive framework for the output feedback control of open-loop stable processes with stable inverse dynamics (Daoutidis and Kravaris, 1992). Furthermore, transparent connections of the derived controller realizations with input/output operator concepts have also been established (Daoutidis and Kravaris, 1992).

In the case of processes with unstable inverse dynamics and/or deadtime (referred to as nonminimumphase processes), research is still at the early stages. In a linear control setting, the customary approach is to employ a factorization of the process transfer function into a minimum-phase and a nonminimum-phase part, with the controller synthesis based on the minimum-phase part, effectively leaving the nonminimum-phase part in open loop. In a nonlinear setting, however, the problem of such a decomposition of the process operator into a minimum-phase and a nonminimum-phase part currently remains an open one [see e.g. Kravaris and Daoutidis (1990)]. Alternatively, nonminimum-phase compensation methods that have been developed rely on state-feedback controller synthesis based on an input/output map that is deadtime-free (Kravaris and Wright, 1989; Wright, 1990) and minimum-phase (Wright and Kravaris, 1992). Despite these results, however, the output feedback control problem for nonminimum-phase processes has not been addressed directly in a comprehensive framework. 
This work deals with nonlinear processes with a state-space description of the form

$$
\dot{x}=f(x)+g(x) u
$$

where $x \in \mathbb{R}^{n}, u \in \mathbb{R}$ denote the state variable vector and the manipulated input, respectively, and $f$ and $g$ denote smooth vector fields on $\mathbb{R}^{n}$. It is assumed that the dynamics of eq. (1) is locally exponentially stable around the nominal equilibrium point. With the above process description we associate an output map of the form $y=h(x)$, where $h$ is a smooth scalar field on $\mathbb{R}^{n}$. We also consider processes with deadtime 0 , for which $y(t)=h(x(t-\theta))$. In the presence of unstable inverse dynamics [or equivalently, unstable zero dynamics in the sense of Byrnes and Isidori, (1985)] and/or deadtime we will say that the process is nonminimum-phase. A deadtime-free process with stable inverse dynamics will be called minimumphase. The purpose of this work is then threefold:

(1) To develop a unified conceptual framework for the control of open-loop stable nonlinear processes (minimum-phase or nonminimumphase).

(2) To derive reduced-order controller realizations for open-loop stable nonminimum-phase processes.

(3) To study the underlying structure of the reduced-order controller realizations.

The first objective is motivated by the inadequacy of the classical error feedback control structure to provide a transparent analysis framework in the presence of deadtime or unstable inverse dynamics. For this reason, a Smith-type abstract operator structure will be introduced that generalizes/unifies the available nonminimum-phase compensation structures in an abstract operator setting and systematically allows a reduction of the control problem for nonminimumphase processes to a control problem for minimumphase processes. Once this goal is accomplished, the controller synthesis problem will be addressed in a state-space framework, through combination of state feedback controllers and state observers. Reduced-order controller realizations will be derived and their underlying structure will be identified and studied. Finally, the proposed control method will be applied to a chemical reaction system that exhibits nonminimum-phase behavior.
THE CLASSICAL ERROR FEEDBACK STRUCTURE AND THE OUTPUT FEEDBACK CONTROL PROBLEM FOR MINIMUM-PHASE PROCESSES

In this section, we briefly review the output feedback control problem for open-loop stable minimumphase processes, within the framework of the classical error feedback structure. Consider the classical error feedback control structure of Fig. 1, where $P$ and $C$ represent nonlinear input/output operators corresponding to the nonlinear process and the classical feedback controller, respectively. In the above setting, it can be easily verified that the following relations hold in terms of compositions of operators:

$$
\begin{aligned}
& y=P C(I+P C)^{-1} y_{s p} \\
& u=C(I+P C)^{-1} y_{s p}
\end{aligned}
$$

where $y_{s p}$ denotes the output set point, and $I$ denotes the identity operator. Setting:

$$
C(I+P C)^{-1}=Q
$$

one obtains a $Q$-parametrization of the controller operator:

$$
C=Q(I-P Q)^{-1}
$$

and the relations of eq. (2) take the form

$$
\begin{aligned}
& \boldsymbol{y}=\boldsymbol{P} \boldsymbol{Q y _ { s p }} \\
& \boldsymbol{u}=Q \boldsymbol{y}_{s p} .
\end{aligned}
$$

Under the assumption of a stable (in an input/output sense) process operator $P$, one can then obtain a transparent characterization of closed-loop stability: the closed-loop system will be stable if and only if the operator $Q$ is stable. Furthermore, imposing a desired closed-loop behavior of the form:

$$
y=R y_{s p}
$$

yields

$$
Q=P^{-1} R
$$

or equivalently

$$
C=P^{-1} R(I-R)^{-1}
$$

for the controller operator. Clearly, if the operator $P^{-1}$ is also stable and causal (i.e. the process is minimum-phase), $R$ can be chosen to be any input/output stable operator.

The above abstract operator treatment provides valuable insights but does not solve the controller

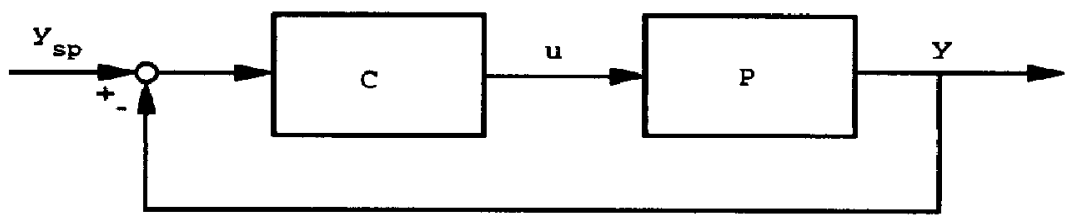

Fig. 1. Classical error feedback control structure. 
synthesis problem because it does not address the realization problem:

- What choice of $R$ guarantees realizability of the contreller operator?

- If the process operator $P$ is given in terms of a differential equation representation, how could one find a differential equation representation for the controller operator?

The above realization problem can be addressed effectively in a state-space framework. In particular, if $R$ is chosen to be a linear operator, the smallest possible order of $\boldsymbol{R}$ that results in a realizable controller operator is equal to the relative order $r$ between $h(x)$ and $u$, i.e. $R$ is of the form

$$
R=\left(\gamma_{r} \frac{\mathrm{d}^{r}}{\mathrm{~d} t^{r}}+\cdots+\gamma_{1} \frac{\mathrm{d}}{\mathrm{d} t}+1\right)^{-1}
$$

where $\gamma_{1}, \ldots, \gamma_{r}$ are adjustable parameters (Daoutidis and $\mathrm{K}$ ravaris, 1992). With the above choice of $R$, unity static gain is automatically enforced in the closedloop system, while input/output stability and desired dynamic performance can be achieved by appropriate choice of the adjustable parameters. Explicit realizations of the controller operator $C=P^{-1} R(I-R)^{-1}$, when $P$ is given in state-space form and $R$ is given by eq. (9), have been derived by Daoutidis and Kravaris (1992) through the combination of input/output lincarizing static state feedback with an open-loop state observer and a linear controller with integral action.

\section{A SMITH-TYPE ABSTRACT OPERATOR STRUCTURE AND THE OUTPUT FEEDBACK CONTROL PROBLEM FOR NONMINIMUM-PHASE PROCESSES}

Referring to the classical error feedback structure and the analysis of the previous section, in the case of nonminimum-phase processes (i.e. when $P^{-1}$ is unstable and/or noncausal), an arbitrary choice of a stable closed-loop operator $R$ will not in general give rise to a stable operator $Q$. In order to deal with this case, and in analogy with the generalized Smith predictor structure for linear nonminimum-phase processes (Ramanathan et al., 1989), we propose here a Smith-type abstract operator structure, which is shown in Fig. 2. The Smith-type abstract operator structure provides valuable insights from an analysis point of view, and allows the reduction of the control problem for nonminimum-phase processes to the one for minimum-phase processes, at a conceptual level; state-space methods must then be used to address the controller realization problem.

The basic idea behind the structure of Fig. 2 is the following: if $\boldsymbol{P}^{*}$ represents a stable operator with stable and causal inverse, one can estimate what the output would have been if $P^{*}$ were the process operator, by adding an appropriate corrective signal (obtained as the difference of the responses of $P^{*}$ and $P$, driven by $u$ ) to the on-line measurement of $y$. Once this auxiliary output $y^{*}$ is estimated, the error between $y_{s p}$ and $y^{*}, e^{*}=y_{s p}-y^{*}$ can be fed to the controller $C^{*}$, which can be synthesized on the basis of $P^{*}$.

More specifically, referring to the structure of Fig. 2, it is straightforward to verify that the following relations between the input and output variables hold, in terms of composition of operators:

$$
\begin{aligned}
y & =P C^{*}\left(I+P^{*} C^{*}\right)^{-1} y_{s p} \\
u & =C^{*}\left(I+P^{*} C^{*}\right)^{-1} y_{s p} \\
y^{*} & =P^{*} C^{*}\left(I+P^{*} C^{*}\right)^{-1} y_{s p} .
\end{aligned}
$$

Assuming that the operators $P^{*}$ and $P^{*-1}$ are stable and causal and that $C^{*}$ is synthesized on the basis of $P^{*}$, i.e.

$$
C^{*}=P^{*-1} R^{*}\left(I-R^{*}\right)^{-1}
$$

for some $R^{*}$, the closed-loop input/output relations take the form

$$
\begin{aligned}
y & =P P^{*-1} R^{*} y_{s p} \\
u & =P^{*-1} R^{*} y_{s p} \\
y^{*} & =R^{*} y_{s p} .
\end{aligned}
$$

Closed-loop stability is then guaranteed for an arbitrary choice of a stable operator $R^{*}$. Recalling the classical error feedback structure, we can easily see that the same result would have been obtained by selecting

$$
C=P^{*-1} R^{*}\left(I-P P^{*-1} R^{*}\right)^{-1}
$$

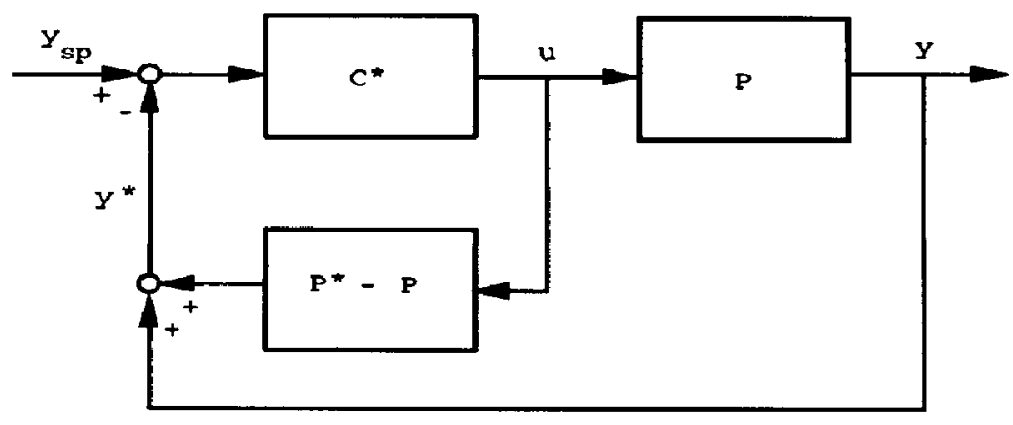

Fig. 2. Smith-type operator structure. 
or, equivalently,

$$
Q=P^{*-1} R^{*}
$$

Clearly, though, the development is more natural and transparent in the framework of the Smith-type structure. An issue that cannot be addressed, however, in the above framework is the choice of $P^{*}$ and $R^{*}$ that

- guarantees unity static gain in the closed-loop system ( $y=v_{s_{p}}$ at steady state).

- optimizes dynamic performance in terms of an appropriate criterion.

Characterizing and imposing the above requirements, as well as deriving the controller realizations, is only possible in a state-space framework. The above issues have been addressed in the context of the Minimumphase Output Predictor method (Wright and Kravaris, 1992) for deadtime-free processes with unstable inverse dynamics and the Nonlinear Smith Predictor method (Wright, 1990) for processes with deadtime and stable inverse dynamics. In what follows we briefly review the basic points from these methods, placing emphasis on their interpretation within the framework of the Smith-type abstract operator structure. First, we review the instrumental notion of statically equivalently outputs (Wright and Kravaris, 1992): two outputs $y_{1}=h_{1}(x)$ and $y_{2}=h_{2}(x)$ are called statically equivalent with respect to $\dot{x}=$ $f(x)+g(x) u$ if $h_{1}(x)=h_{2}(x) \forall x \in E$, where $E$ is the equilibrium set

$$
E=\left\{x \in \mathbb{R}^{n} \mid \exists u \in \mathbb{R}: f(x)+g(x) u=0\right\} .
$$

Consider now a nonlinear process $(P)$ with a statespace description of the form

$$
\begin{aligned}
& \dot{x}=f(x)+g(x) u \\
& y=h(x)
\end{aligned}
$$

whose zero dynamics is unstable. Following the Minimum-phase Output Predictor method, the following choices are made for $P^{*}$ and $R^{*}$ :

(1) $P^{*}$ has the form

$$
\begin{aligned}
\dot{x} & =f(x)+g(x) u \\
y^{*} & =h^{*}(x)
\end{aligned}
$$

where $h^{*}(x)$ is chosen so that

- $y^{*}=h^{*}(x)$ is a minimum-phase output to the dynamics $\dot{x}=f(x)+g(x) u$,

- $h^{*}(x)$ is statically equivalent to $h(x)$ (to guarantee unity static gain in the closed-loop system).

(2) $R^{*}$ is a linear closed-loop operator of the smallest order that guarantees a realizable $P^{*-1} R^{*}$, i.e.

$$
R^{*}=\left(\gamma_{r^{*}} \frac{\mathrm{d}^{r^{*}}}{\mathrm{~d} t^{r^{*}}}+\cdots+\gamma_{1} \frac{\mathrm{d}}{\mathrm{d} t}+1\right)^{-1}
$$

where $r^{*}$ is the relative order between $h^{*}(x)$ and $u$, and $\gamma_{1}, \ldots, \gamma_{r^{*}}$ are adjustable parameters.
Based on the above choices, one can readily obtain a realization of the controller operator $C^{*}$ $=P^{*-1} R^{*}\left(I-R^{*}\right)^{-1}$ using the results in Daoutidis and Kravaris (1992) for minimum-phase processes, while a realization of $P^{*}-P$ is readily obtained from the realizations of $P^{*}$ and $P$, completing the controller synthesis.

Remark 1: The choices of $P^{*}$ and $R^{*}$ described above are in complete agreement with linear transfer function methods that are based on the Bode factorizations of the process transfer function. With these choices, instead of "tuning" the operators $P^{*}$ and $R^{*}$, one must tune the output function $h^{*}(x)$ and the parameters $\gamma_{1}, \ldots, \gamma_{r^{*}}$

Remark 2: For the above choices, the operator $P^{*}-P$ becomes the Minimum-phase Output Predictor, and the Smith-type abstract operator structure reduces to the Minimum-phase Output Predictor structure.

Remark 3: To complete the design, an optimal choice of $h^{*}$ (in terms of some performance criterion) must be made from the set of minimum-phase outputs that are statically equivalent to $h$. A Lagrangian formulation of the ISE optimization problem as well as analytical expressions for the ISE-optimal $h^{*}$ for special cases are available in Wright and $K$ ravaris (1992).

Consider now a nonlinear process $(P)$ with deadtime $\theta$ and a state-space description of the form

$$
\begin{aligned}
& \dot{x}=f(x)+g(x) u \\
& y=h(x(t-\theta)) .
\end{aligned}
$$

Assume that the zero dynamics associated with $h(x)$ is stable. Then, following the Nonlinear Smith Predictor method, the following choices are made for $P^{*}$ and $R^{*}$ :

(1) $P^{*}$ is the deadtime-free part of $P$ :

$$
\begin{aligned}
\dot{x} & =f(x)+g(x) u \\
y^{*} & =h(x)
\end{aligned}
$$

i.e. $y^{*}$ represents what the output would have been if there were no deadtime.

(2) $R^{*}$ is a linear closed-loop operator of the smallest order that guarantees a realizable $P^{*-1} R^{*}$, i.e.

$$
R^{*}=\left(\gamma_{r} \frac{\mathrm{d}^{r}}{\mathrm{~d} t^{r}}+\cdots+\gamma_{1} \frac{\mathrm{d}}{\mathrm{d} t}+1\right)^{-1}
$$

where $r$ is the relative order between $h(x)$ and $u$, and $/ \gamma_{1}, \ldots, \gamma_{r}$ are adjustable parameters.

Similarly to the previous case, one can readily obtain a realization of the controller operator $C^{*}=$ $P^{*-1} R^{*}\left(I-R^{*}\right)^{-1}$ and a realization of $P^{*}-P$ to complete the controller synthesis. 
Remark 4: For the above choices, the operator $P^{*}-\boldsymbol{P}$ becomes the Nonlinear Smith Predictor, and the Smith-type abstract operator structure reduces to the Nonlinear Smith Predictor structure.

Remark 5: For the above choices of $P^{*}$ and $R^{*}$, unity static gain is automatically enforced in the closedloop system, while, in the limit as the roots of $\gamma_{r} s^{r}+\cdots+\gamma_{1} s+1$ tend to negative infinity, ISEoptimal response to step changes in $y_{s p}$ is obtained.

Finally, let us consider the more general case of a nonlinear process $(P)$ with deadtime $\theta$ and a statespace description of the form

$$
\begin{aligned}
& \dot{x}=f(x)+g(x) u \\
& y=h(x(t-\theta))
\end{aligned}
$$

for which the zero dynamics associated with $h(x)$ is unstable. Referring to the Smith-type abstract operator structure, the following choice of $P^{*}$ and $R^{*}$ is then clearly justified:

(1) $P^{*}$ has the form

$$
\begin{aligned}
\dot{x} & =f(x)+g(x) u \\
y^{*} & =h^{*}(x)
\end{aligned}
$$

where $h^{*}(x)$ is chosen so that

- $y^{*}=h^{*}(x)$ is a minimum-phase output to the dynamics $\dot{x}=f(x)+g(x) u$,

- $h^{*}(x)$ is statically equivalent to $h(x)$.

(2) $R^{*}$ is a linear closed-loop operator of the smallest order that guarantees a realizable $P^{*-1} R^{*}$, i.e.

$$
R^{*}=\left(\gamma_{r^{*}} \frac{\mathrm{d}^{r^{*}}}{\mathrm{~d} t^{r^{*}}}+\cdots+\gamma_{1} \frac{\mathrm{d}}{\mathrm{d} t}+1\right)^{-1}
$$

where $r^{*}$ is the relative order between $h^{*}(x)$ and $u$, and $\gamma_{1}, \ldots, \gamma_{r^{*}}$ are tunable parameters.

Similarly to the two previous cases, based on the above choices the controller synthesis problem reduces to the one for a minimum-phase process $\left(P^{*}\right)$ and can be addressed in a state-space framework.

Remark 6: In the case of a minimum-phase nonlinear process $(P)$ with a state-space description of the form

$$
\begin{aligned}
& \dot{x}=f(x)+g(x) u \\
& y=h(x)
\end{aligned}
$$

the natural choice of $P^{*}=P$ reduces the Smith-type abstract operator structure to the classical error feedback control structure of Fig. 1.

To conclude this section, the key advantages of the proposed Smith-type operator structure are:

(1) It provides a unified conceptual framework for interpreting available minimum- and nonminimum-phase compensation structures, as well as addressing a more general nonminimum-phase compensation problem.
(2) It provides a transparent analysis framework where the issue of closed-loop stability can be casily characterized for nonminimum-phase processes.

(3) It allows a natural reduction of the controller synthesis problem for nonminimum-phase processes to a controller synthesis problem for minimum-phase processes, without resorting to any decomposition of the process operator.

The next section will provide an answer to the controller realization problem.

\section{OUTPUT FEEDBACK CONTROLLER SYNTHESIS FOR NONMINIMUM-PHASE NONLINEAR PROCESSES}

Once the choices described in the previous section have been made, one can formulate the controller synthesis problem and derive output feedback controller realizations on the basis of $P^{*}$ and $R^{*}$. The basic controller synthesis result of the paper will be summarized in the theorem that follows, where a reduced-order controller realization will be derived that induces a desired closed-loop input/output behavior for a process with deadtime and unstable inverse dynamics. Reduced-order controller realizations for the first two cases discussed in the previous section will then result naturally.

Theorem 1: Consider a nonlinear process (P) with deadtime $\theta$ and a state-space description of the form

$$
\begin{aligned}
& \dot{x}=f(x)+g(x) u \\
& y=h(x(t-\theta)) .
\end{aligned}
$$

Consider, also, an output map $\mathrm{h}^{*}$ which is statically equivalent to $\mathrm{h}$ and such that the zero dynamics associated with $\mathrm{y}^{*}=\mathrm{h}^{*}(\mathrm{x})$ is locally exponentially stable around the equilibrium point. Denote by $\mathrm{r}^{*}$ the relative order between $\mathrm{h}^{*}(\mathrm{x})$ and $\mathrm{u}$. Then, the dynamic system $\dot{w}=f(w)+g(w)$

$$
\times \frac{e+h(w(t-\theta))-h^{*}(w)-\sum_{k=1}^{r^{*}} \gamma_{k} L_{f}^{k} h^{*}(w)}{\gamma_{r} L_{\theta} L_{f}^{r^{*}-1} h^{*}(w)}
$$

$u=\frac{e+h(w(t-\theta))-h^{*}(w)-\sum_{k=1}^{* *} \gamma_{k} L_{f}^{k} h^{*}(w)}{\gamma_{r *} L_{a} L_{f}^{*-1} h^{*}(w)}$

represents a controller realization that

- induces the input/output dynamics

$$
\gamma_{r^{*}} \frac{\mathrm{d}^{r^{*}}\left[h^{*}(x)\right]}{\mathrm{d} t^{r^{*}}}+\cdots+\gamma_{1} \frac{\mathrm{d}\left[h^{*}(x)\right]}{\mathrm{d} t}+h^{*}(x)=y_{s p}
$$

with $\mathrm{y}=\mathrm{y}_{\mathrm{sp}}$ at steady state, and

- guarantees internal stability of the closed-loop system, locally around the equilibrium point, provided that 
the process dynamics is locally exponentially stable arotund the equilibrium point

- the polynomial $1+\gamma_{1} s+\cdots+\gamma_{r} s^{r}$ is Hurwitz.

Proof: Referring to the Smith-type abstract operator structure of Fig. 2, and for the choice of $P^{*}$ and $R^{*}$ described in the previous section, the following realizations for the various components of the structure can be easily obtained.

$P^{*}-P$ :

$$
\begin{aligned}
\psi & =f(\psi)+g(\psi) u \\
\delta y & =h^{*}(\psi)-h(\psi(t-\theta)) .
\end{aligned}
$$

$C^{*}=P^{*-1} R^{*}\left(I-R^{*}\right)^{-1}$ :

$\dot{\xi}=\left[\begin{array}{cccccc}0 & 1 & 0 & \cdots & 0 & 0 \\ 0 & 0 & 1 & \cdots & 0 & 0 \\ 0 & 0 & 0 & \cdots & 0 & 0 \\ \vdots & \vdots & \vdots & \ddots & \vdots & \vdots \\ 0 & 0 & 0 & \cdots & 0 & 1 \\ 0 & -\frac{\gamma_{1}}{\gamma_{r^{*}}} & -\frac{\gamma_{2}}{\gamma_{r^{*}}} & \cdots & -\frac{\gamma_{r^{*}-2}}{\gamma_{r^{*}}} & -\frac{\gamma_{r^{*}-1}}{\gamma_{r^{*}}}\end{array}\right] \xi+\left[\begin{array}{c}0 \\ 0 \\ 0 \\ \vdots \\ 0 \\ \frac{1}{\gamma_{r^{*}}}\end{array}\right] e^{*}$

$\dot{w}=f(w)+g(w) \frac{\xi_{1}+e^{*}-h^{*}(w)-\sum_{k=1}^{* *} \gamma_{k} L_{f}^{k} h^{*}(w)}{\gamma_{r^{*}} L_{\theta} L_{f}^{r^{*}-1} h^{*}(w)}$

$u=\frac{\xi_{1}+e^{*}-h^{*}(w)-\sum_{k=1}^{* *} \gamma_{k} L_{f}^{k} h^{*}(w)}{\gamma_{r^{*}} L_{g} L_{f}^{*-1} h^{*}(w)}$

where

$$
e^{*}=y_{s p}-y-\delta y
$$

and the realization of eq. (29) was obtained following Daoutidis and Krayaris (1992). Combining the above realizations, the state-space realization of the controller takes the form

$\dot{\xi}=\left[\begin{array}{cccccc}0 & 1 & 0 & \cdots & 0 & 0 \\ 0 & 0 & 1 & \cdots & 0 & 0 \\ 0 & 0 & 0 & \cdots & 0 & 0 \\ \vdots & \vdots & \vdots & \ddots & \vdots & \vdots \\ 0 & 0 & 0 & \cdots & 0 & 1 \\ 0 & -\frac{\gamma_{1}}{\gamma_{r^{*}}} & -\frac{\gamma_{2}}{\gamma_{r^{*}}} & \cdots & -\frac{\gamma_{r^{*}-2}}{\gamma_{r^{*}}} & -\frac{\gamma_{r^{*}-1}}{\gamma_{r^{*}}}\end{array}\right] \xi+\left[\begin{array}{c}0 \\ 0 \\ 0 \\ \vdots \\ 0 \\ \frac{1}{\gamma_{r^{*}}}\end{array}\right]\left\{e-h^{*}(\psi)+h(\psi(t-\theta))\right\}$

$\dot{w}=f(w)+g(w) \frac{\xi_{1}+\varepsilon-h^{*}(\psi)+h(\psi(t-\theta))-h^{*}(w)-\sum_{k=1}^{r^{*}} \gamma_{k} L_{f}^{k} h^{*}(w)}{\gamma_{r *} L_{\theta} L_{f}^{r^{*}-1} h^{*}(w)}$

$\dot{\psi}=f(\psi)+g(\psi) \frac{\xi_{1}+e-h^{*}(\psi)+h(\psi(t-\theta))-h^{*}(w)-\sum_{k=1}^{* *} \gamma_{k} L_{f}^{k} h^{*}(w)}{\gamma_{r^{*}} L_{g} L_{f}^{r^{*}-1} h^{*}(w)}$

$u=\frac{\xi_{1}+e-h^{*}(\psi)+h(\psi(t-\theta))-h^{*}(w)-\sum_{k-1}^{r_{1}^{*}} \gamma_{k} L_{f}^{k} h^{*}(w)}{\gamma_{r^{*}} L_{g} L_{f}^{r^{*}-1} h^{*}(w)}$.

Clearly, if the controller states are initialized consistently $[w(0)=\psi(0)]$, we will have $w(t)=\psi(t)$ for all 
$t$, and the above realization reduces to

$\xi=\left[\begin{array}{cccccc}0 & 1 & 0 & \cdots & 0 & 0 \\ 0 & 0 & 1 & \cdots & 0 & 0 \\ 0 & 0 & 0 & \cdots & 0 & 0 \\ \vdots & \vdots & \vdots & \ddots & \vdots & \vdots \\ 0 & 0 & 0 & \cdots & 0 & 1 \\ 0 & -\frac{\gamma_{1}}{\gamma_{r^{*}}} & -\frac{\gamma_{2}}{\gamma_{r^{*}}} & \cdots & -\frac{\gamma_{r^{*}-2}}{\gamma_{r^{*}}} & -\frac{\gamma_{r^{*}-1}}{\gamma_{r^{*}}}\end{array}\right] \xi+\left[\begin{array}{c}0 \\ 0 \\ 0 \\ \vdots \\ 0 \\ \frac{1}{\gamma_{r^{*}}}\end{array}\right]\left\{e-h^{*}(w)+\boldsymbol{h}(w(t-\theta))\right\}$

$\dot{w}=f(w)+g(w) \frac{\xi_{1}+e-h^{*}(w)+h(w(t-\theta))-h^{*}(w)-\sum_{k=1}^{r^{*}} \gamma_{k} L_{f}^{*} h^{*}(w)}{\gamma_{r^{*}} L_{g} L_{f}^{r^{*}-1} h^{*}(w)}$

$u=\frac{\xi_{1}+e-h^{*}(w)+h(w(t-0))-h^{*}(w)-\sum_{k=1}^{* *} \gamma_{k} L_{f}^{k} h^{*}(w)}{\gamma_{r^{*}} L_{g} L_{f}^{r^{*}-1} h^{*}(w)}$.

Furthermore, if

$$
\xi_{i}(0)=L_{J}^{i-1} h^{*}(w)(0), \quad i=1, \ldots, r^{*}
$$

it follows that

$$
\xi_{i}=L_{f}^{i-1} h^{*}(w), \quad i=1, \ldots, r^{*}
$$

i.e. $\xi_{1}=h^{*}(w)$. Substituting this relation to eq. (32), we obtain the controller realization of eq. (26). The closed-loop system under the controller of eq. (26)

takes the form

$$
\begin{aligned}
& \dot{x}=f(x)+g(x) \frac{y_{s p}-h(x(t-\theta))+h(w(t-\theta))-h^{*}(w)-\sum_{k=1}^{r_{1}^{*}} \gamma_{k} L_{f}^{k} h^{*}(w)}{\gamma_{r^{*}} L_{\theta} L_{f}^{*-1} h^{*}(w)} \\
& \dot{w}=f(w)+g(w) \frac{y_{s p}-h(x(t-\theta))+h(w(t-\theta))-h^{*}(w)-\sum_{k=1}^{*} \gamma_{k} L_{f}^{k} h^{*}(w)}{\gamma_{r} L_{g} L_{f}^{r^{*-1}} h^{*}(w)} \\
& y=h(x(t-\theta)) .
\end{aligned}
$$

Under the assumptions of the theorem, Liapunov's first theorem can be employed to infer local asymptotic stability in the sense of Liapunov around the equilibrium in the unforced closed-loop system. (The above analysis is greatly facilitated by transforming the closed-loop system in normal form coordinates; details are omitted for brevity.)

Furthermore, under consistent initialization of $x$ and $w[x(0)=w(0)]$, eq. (35) reduces to

$$
\begin{aligned}
& \dot{x}=f(x)+g(x) \frac{y_{s p}-h^{*}(x)-\sum_{k=1}^{* *} \gamma_{k} L_{f}^{k} h^{*}(x)}{\gamma_{r}^{*} L_{g} L_{f}^{* * 1} h^{*}(x)} \\
& y=h(x(t-\theta))
\end{aligned}
$$

which (as can be easily verified) results in the following input/output dynamics:

$$
\gamma_{r^{*}} \frac{\mathrm{d}^{r^{*}}\left[h^{*}(x)\right]}{\mathrm{d} t^{r^{*}}}+\cdots+\gamma_{1} \frac{\mathrm{d}\left[h^{*}(x)\right]}{\mathrm{d} t}+h^{*}(x)=y_{s p}
$$

Clearly, at steady state, $h^{*}(x)=y_{s p}$, and by the static equivalence of $h$ and $h^{*}, h(x)=y_{s p}$ as well, which concludes the proof.
The above theorem provides a comprehensive result that can be applied to any open-loop stable nonlinear process, with or without deadtime, with stable or unstable zero dynamics. The controller realization depends on the choice of an appropriate output map $h^{*}$ that optimizes a given performance criterion.

Remark 7: In the case of a deadtime-free process with a state-space description of the form of eq. (15) and unstable zero dynamics, the controller realization of eq. (26) takes the form

$$
\begin{aligned}
& \dot{w}=f(w)+g(w) \frac{e+h(w)-h^{*}(w)-\sum_{k-1}^{r^{*}} \gamma_{k} L_{f}^{k} h^{*}(w)}{\gamma_{r^{*}} L_{g} L_{f}^{r^{*}-1} h^{*}(w)} \\
& u=\frac{e+h(w)-h^{*}(w)-\sum_{k=1}^{r^{*}} \gamma_{k} L_{f}^{k} h^{*}(w)}{\gamma_{r^{*}} L_{g} L_{f}^{r^{*}-1} h^{*}(w)}
\end{aligned}
$$

and the resulting input/output dynamics

$$
\gamma_{r^{*}} \frac{\mathrm{d}^{r^{*}}\left[h^{*}(x)\right]}{\mathrm{d} r^{*}}+\cdots+\gamma_{1} \frac{\mathrm{d}\left[h^{*}(x)\right]}{\mathrm{d} t}+h^{*}(x)=y_{s p}
$$


Remark 8: In the case of a process with deadtime and a state-space description of the form of eq. (18) for which the zero dynamics associated with $h(x)$ is stable, and for the natural choice of $h^{*}=h$, the controller realization of eq. (26) becomes
(1) It goes through the model output map to generate the model output, which is then added to the error signal to generate $v=$ (set point) - (process output) + (model output).

$$
\begin{aligned}
& \dot{w}=f(w)+g(w) \frac{e+h(w(t-\theta))-h(w)-\sum_{k=1}^{r} \gamma_{k} L_{f}^{k} h(w)}{\gamma_{r} L_{\theta} L_{f}^{r-1} h(w)} \\
& u=\frac{e+h(w(t-\theta))-h(w)-\sum_{k=1}^{r} \gamma_{k} L_{f}^{k} h(w)}{\gamma_{r} L_{\theta} L_{f}^{r-1} h(w)}
\end{aligned}
$$

and the induced input/output behavior

$$
\gamma_{r} \frac{d^{r} y}{d t^{r}}+\cdots+\gamma_{1} \frac{d y}{d t}+y=y_{s p}(t-\theta)
$$

Remark 9: Finally, in the case of a minimum-phase nonlinear process with a state-space description of the form of eq. (24), and for the natural choice of $h^{*}=h$, the controller realization of eq. (26) becomes

$$
\begin{aligned}
\dot{w} & =f(w)+g(w) \frac{e-\sum_{k=1}^{r} \gamma_{k} L_{f}^{k} h(w)}{\gamma_{r} L_{g} L_{f}^{r-1} h(w)} \\
u & =\frac{e-\sum_{k=1}^{r} \gamma_{k} L_{f}^{k} h(w)}{\gamma_{r} L_{\theta} L_{f}^{r-1} h(w)}
\end{aligned}
$$

which, as expected, is identical to the reduced-order controller realization derived in Daoutidis and Kravaris (1992), inducing the closed-loop input/ output behavior

$$
\gamma_{r} \frac{d^{r} y}{d t^{r}}+\cdots+\gamma_{1} \frac{d y}{d t}+y=y_{s p}
$$

The model state feedback controller

The controller realization of eq. (26) as well as the controllers of eqs (38) (40) and (42) derived for the various special cases, share a common underlying structure which is depicted in Fig. 3. Referring to this structure, we observe that the process model $\dot{w}=f(w)+g(w) w$ is simulated on-line, with the model state $w$ representing an on-line estimate of the true process state $x$. The model state $w$ is then utilized in two ways:
(2) It is fed back via the static state feedback law

$$
u=\frac{v-h^{*}(w)-\sum_{k=1}^{*} \gamma_{k} L_{f}^{k} h^{*}(w)}{\gamma_{r^{*}} L_{g} L_{f}^{* * 1} h^{*}(w)}
$$

which is an input/output linearizing feedback law for the output map $h^{*}$.

The controller structure of Fig. 3 and its key features were identified and discussed in Kravaris and Daoutidis (1992). The same structure was subsequently used by Coulibaly et al. (1992) in a linear context for the purpose of handling of input constraints and was compared with standard IMC- and DMC-type implementations. The structure of Fig. 3 is also inherent in MAC-type nonlinear and model predictive control strategies (Soroush and Kravaris, $1992 a, b)$. A similar structure incorporating disturbance measurements was also identified in Daoutidis and Alhumaizi (1993) within a feedforward/output feedback synthesis framework.

In what follows, the properties of the controller structure of Fig. 3 will be analyzed in a more general setting. We will use the name "model state feedback controller" for the corresponding controller, as suggested by Coulibaly et al. (1992), since it captures the essence of the internal structure of the controller: instead of feeding back just the model output, the entire model state is fed back.

Figure 4 refers to processes with a state-space model of the form of eq. (1) and depicts the structure of a general model state feedback controller, whose static state feedback component has the general form

$$
u=p(w)+q(w) v
$$

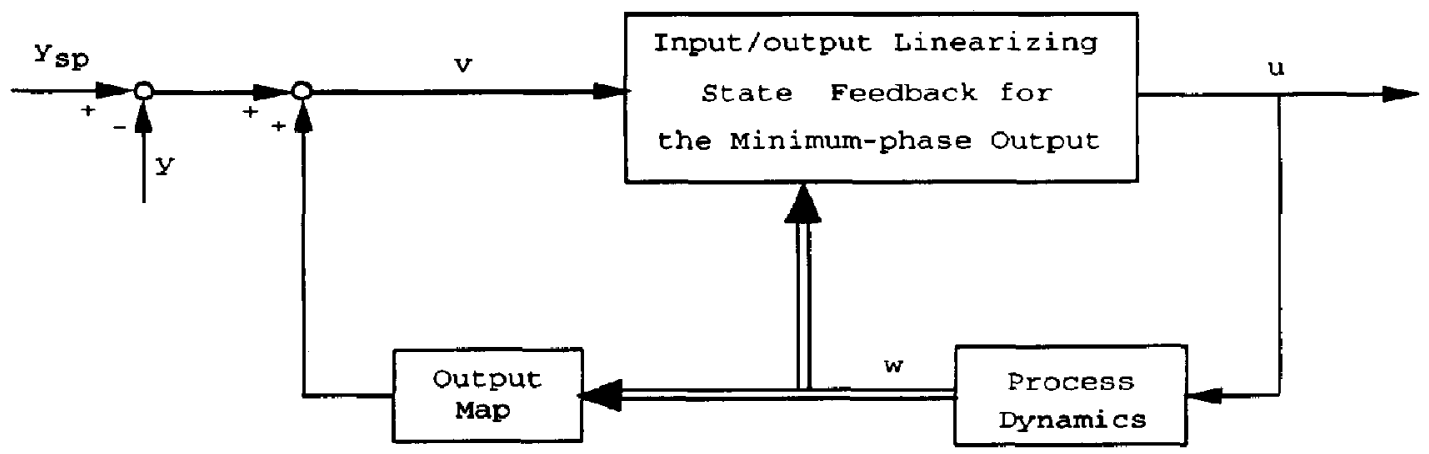

Fig. 3. Structure of reduced-order output feedback controllers. 


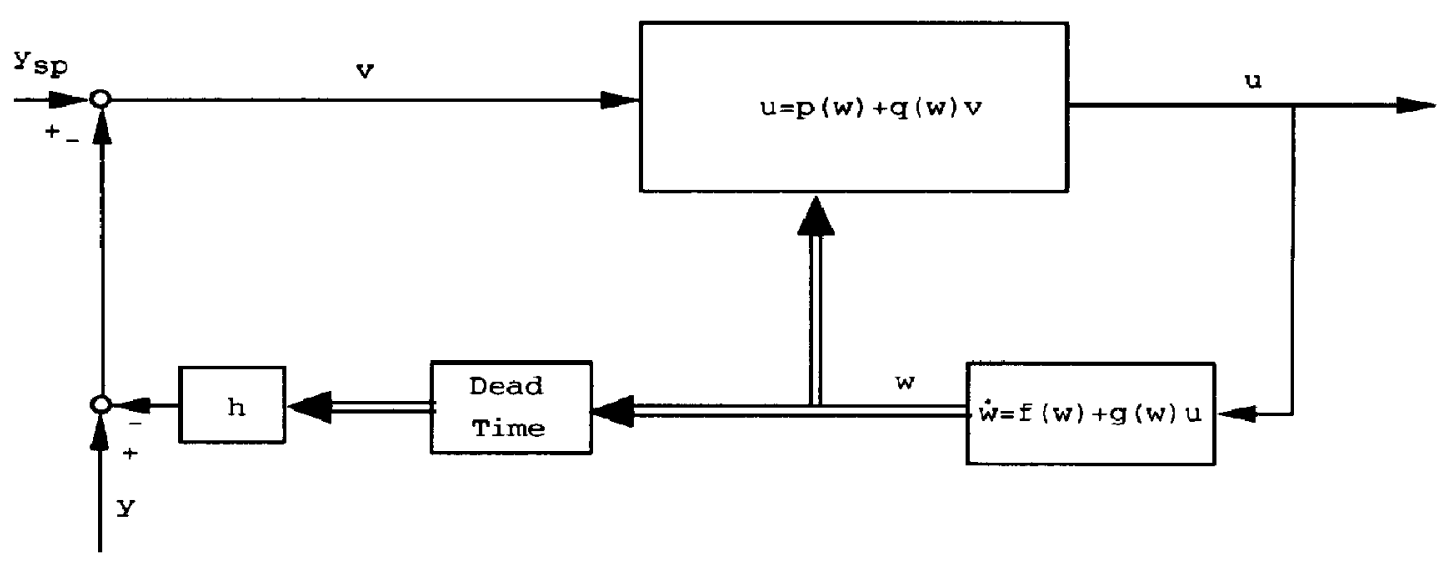

Fig. 4. Structure of a general model state feedback controller.

instead of the specific form of eq. (14). The general form of the model state feedback controller becomes then

$$
\begin{aligned}
\dot{w}= & f(w)+g(w)\{p(w)+g(w) h(w(t-\theta))\} \\
& +g(w) q(w)\left(y_{s p}-y\right) \\
u= & p(w)+q(w) h(w(t-\theta))+q(w)\left(y_{s p}-y\right) .
\end{aligned}
$$

Referring to Fig. 4, the key features of this controller can be easily identified. When the process dynamics is perfectly modeled and initialized, the model state $w(t)$ will match the process state $x(t)$. If in addition, the output map is perfectly known, the model output will match the process output $y$ as well; in this case $v=y_{s p}$ and the controller action will be the same as if all the states were measured on-line and the static state feedback

$$
u=p(x)+q(x) y_{s p}
$$

were used.

The same conclusion can be drawn by combining the controller of eq. (45) with the process dynamics of eq. (25):

$$
\begin{aligned}
& \dot{x}=f(x)+g(x) u \\
& y=h(x(t-\theta)) .
\end{aligned}
$$

The resulting closed-loop system is given by

$$
\begin{aligned}
\dot{w}= & f(w)+g(w)(p(w)+q(w)\{h(w(t-\theta)) \\
& -h(x(t-\theta))\})+g(w) q(w) y_{s p} \\
\dot{x}= & f(x)+g(x)(p(w)+q(w)\{h(w(t-\theta)) \\
& -h(x(t-\theta))\})+g(w) q(w) y_{s p} \\
y= & h(x(t-\theta)) .
\end{aligned}
$$

We observe that both $w$ and $x$ follow the same dynamics $\dot{w}=f(w)+g(w) u$ and $\dot{x}=f(x)+g(x) u$, driven by the same input:

$$
\begin{aligned}
u= & p(w)+q(w)\{h(w(t-\theta)) \\
& -h(x(t-\theta))\}+q(w) y_{s p} .
\end{aligned}
$$

Therefore, under consistent initialization, $w(t)=x(t)$ for all times, and the closed-loop dynamics simplifies to

$$
\dot{x}=f(x)+g(x) p(x)+g(x) q(x) y_{s p}
$$

which is exactly the closed-loop dynamics that would have been obtained if the static state feedback:

$$
u=p(x)+g(x) q(x) y_{s p}
$$

had been applied to the process. It is important to note that the reduction of the dimensionality of the closed-loop dynamics described above happened after the matching of the model states with the process states. Expressed in a linear control terminology, this reduction happened after "zero-pole cancellations at the process modes". The above property justifies the instrumental role of the assumption of open-loop stability of the process in the derivation of the model state feedback controller.

In summary, the key property of the model state feedback controller is that it allows the reduction of a dynamic output feedback synthesis problem into a static state feedback synthesis problem, under the assumption of open-loop stability of the process. The presence of modeling error does not alter the synthesis formula for the control law, but will influence the choice of the tunable parameters in view of the wellknown trade-offs between performance and robustness.

\section{Integral action}

In this subsection, we will conclude the theoretical part of the paper by addressing the important issue of integral action of the controller realization of theorem 1 [and consequently the controllers of eqs (38), (40) and (42) derived for the various special cases.] To this end, an important question that arises is what is the precise meaning of integral action in a nonlinear context. Because the significance of integral action is connected with steady-state properties of the closed-loop system, it seems natural to define 
integral action on the basis of steady-state considerations. In a linear context, integral action is associated with infinite static gain or, equivalently, the inverse of the dynamic system having zero static gain. This observation motivates the following generalization of the notion of integral action in a nonlinear setting-

Definition. The dynamic system

$$
\begin{gathered}
\dot{w}=\phi(w)+\psi(w) e \\
u=\pi(w)+m(w) e
\end{gathered}
$$

where $\phi(w), \psi(w)$ are vector fields of appropriate dimension and $\pi(\mathrm{w}), \omega(\mathrm{w})$ are scalar fields, is said to possess integral action if its inverse:

$$
\begin{aligned}
& \dot{w}=\phi(w)-\frac{\pi(w)}{\omega(w)} \psi(w)+\frac{1}{\omega(w)} \psi(w) u \\
& e=-\frac{\pi(w)}{\omega(w)}+\frac{1}{\omega(w)} u
\end{aligned}
$$

has zero static gain, i.e. every pair $\left(\mathrm{u}_{\mathrm{s}}, \mathrm{w}_{\mathrm{s}}\right)$ that satisfies

$$
\phi\left(w_{s}\right)-\frac{\pi\left(w_{s}\right)}{\omega\left(w_{s}\right)} \psi\left(w_{s}\right)+\frac{1}{\omega\left(w_{s}\right)} \psi\left(w_{s}\right) u_{s}=0
$$

also satisfies

$$
-\pi\left(w_{s}\right)+u_{s}=0 .
$$

Considering now the controller realization of eq. (26), we easily obtain the following realization of its inverse:

$$
\begin{aligned}
\dot{w}= & f(w)+g(w) u \\
e= & -h(w(t-\theta))+h^{*}(w) \\
& \quad+\sum_{k=1}^{r^{*}} \gamma_{k} L_{f}^{k} h^{*}(w)+\gamma_{* *} L_{g} L_{f}^{*-1} h^{*}(w) u .
\end{aligned}
$$

We observe that the dynamics of the controller inverse coincide with the dynamics of the process model, and, therefore, their steady-states coincide as well. Hence, based on the previous definition of integral action and in view of the static equivalence of $h$ and $h^{*}$, the controller of eq. (26) will possess integral action if the following relation is satisfied:

$$
\sum_{k=1}^{r *} \gamma_{k} L_{f}^{k} h^{*}\left(w_{s}\right)+\gamma_{r} L_{g} L_{f}^{r^{*}-1} h^{*}\left(w_{s}\right) u_{s}=0
$$

where $\left(u_{s}, w_{s}\right)$ is a steady-state pair of

$$
\dot{w}=f(w)+g(w) u .
$$

A straightforward argument based on the definition of relative order can be used to show that

$$
\begin{aligned}
& L_{f}^{k} h^{*}\left(w_{s}\right)=0, \quad k=1, \ldots, r^{*}-1 \\
& L_{f}^{* *} h^{*}\left(w_{s}\right)=-L_{g} L_{f}^{* * 1} h^{*}\left(w_{s}\right) u_{s} .
\end{aligned}
$$

Therefore, eq. (49) indeed holds and the controller of eq. (26) possesses integral action.

\section{NONMINIMUM-PHASE BEHAVIOR IN A CLASS OF CHEMICAL REACTION SYSTEMS}

The motivation for this section arises from the fact that quite often exothermic chemical reactors operate with cold feed, which can give rise to inverse response characteristics (and thus, nonminimum-phase behavior) in temperature control problems [e.g. Juba and Hamer (1986) and McLellan et al., (1990)]. More specifically, for a continuous exothermic reactor where the inlet stream flowrate is used to control the reactor temperature, a positive step change in the inlet flowrate will cause an initial decrease in the reactor temperature; however, the reaction rate will eventually increase, causing the reactor temperature to increase as well. Similarly, a negative step change in the inlet flowrate will cause an initial increase in the reactor temperature, but eventually the reaction effects will dominate causing a decrease in the reactor temperature. Motivated by the above, in this section we illustrate the application of the proposed nonlinear control methodology to a continuous stirred tank reactor (CSTR) that exhibits such behavior.

More specifically, we consider an ideal CSTR, where a solution stream consisting of pure $A$ at concentration $C_{A O}$ and temperature $T_{0}$ enters the reactor, and the exothermic reaction

$$
\mathbf{A} \rightarrow \mathbf{B}
$$

takes place. A cooling jacket maintained at a temperature $T_{j}$ is used for heat removal. The effluent stream leaves the reactor at concentrations $c_{A}, c_{B}$ and temperature $T$. Under standard assumptions, the mass and energy balance describing the dynamic behavior of the process take the form

$$
\begin{gathered}
\frac{\mathrm{d} c_{A}}{\mathrm{~d} t}=\frac{F}{V}\left(c_{A 0}-c_{A}\right)-k(T) c_{A} \\
\frac{\mathrm{d} T}{\mathrm{~d} t}=\frac{F}{V}\left(T_{0}-T\right)+\gamma k(T) c_{A}-\alpha\left(T-T_{j}\right)
\end{gathered}
$$

where

$$
\begin{gathered}
\boldsymbol{k}(T)=k_{0} e^{-\boldsymbol{E} / \boldsymbol{R} T} \\
\gamma=-\frac{\Delta H}{\rho C_{p}} \\
\alpha=\frac{U A}{V \rho C_{\mathrm{D}}}
\end{gathered}
$$

and the values of the various process parameters are shown in Table 1. Motivated by the discussion in the beginning of this section, we consider a temperature control problem for this process, using as manipulated input the inlet flowrate $F$.

\section{Equilibrium diagram}

The steady-state solutions for the above process can be found by setting the right-hand side of eq. (50) equal to 0 . Eliminating the flowrate $F$ from the resulting algebraic equations, we obtain the following rela- 
Table 1. Process parameters

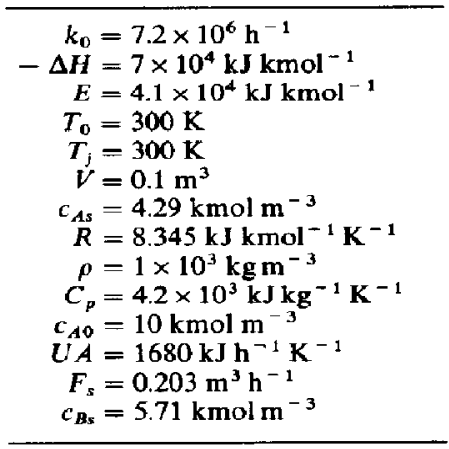

tion that has to be satisfied at steady state:

$$
\begin{aligned}
c_{A}^{2}-\left\{c_{A 0}+\frac{\left(T-T_{0}\right)}{\gamma}\right. & {\left.\left[\frac{\left(T-T_{j}\right)}{\left(T-T_{0}\right)} \frac{\alpha}{k(T)}-1\right]\right\} c_{A} } \\
& +\frac{\alpha}{k(T)} \frac{\left(T-T_{j}\right)}{\gamma} c_{A 0}=0 .
\end{aligned}
$$

Equation (51) is a quadratic equation in $c_{A}$. It is easily verified that if the following conditions are satisfied:

$$
\begin{gathered}
T-T_{j}>0 \\
\left\{c_{A 0}+\frac{\left(T-T_{0}\right)}{\gamma}\left[\frac{\left(T-T_{j}\right)}{\left(T-T_{0}\right)} \frac{\alpha}{k(T)}-1\right]\right\}>0 \\
\left\{c_{A 0}+\frac{\left(T-T_{0}\right)}{\gamma}\left[\frac{\left(T-T_{j}\right)}{\left(T-T_{0}\right)} \frac{\alpha}{k(T)}-1\right]\right\}^{2} \\
-4 \frac{\alpha}{k(T)} \frac{\left(T-T_{j}\right)}{\gamma} c_{A 0} \geqslant 0
\end{gathered}
$$

eq. (51) will give rise to positive real roots. The root of the equation:

$$
\begin{aligned}
\left\{c_{A O}+\frac{\left(T-T_{0}\right)}{\gamma}[\right. & \left.\left.\frac{\left(T-T_{j}\right)}{\left(T-T_{0}\right)} \frac{\alpha}{k(T)}-1\right]\right\}^{2} \\
& -4 \frac{\alpha}{k(T)} \frac{\left(T-T_{j}\right)}{\gamma} c_{A O}=0
\end{aligned}
$$

determines a temperature $T_{\max }$ that gives rise to a double root in $c_{A}$ :

$$
\begin{aligned}
c_{A} \mid T_{T=T_{\max }=\frac{1}{2}} & \left\{c_{A 0}+\frac{\left(T-T_{0}\right)}{\gamma}\right. \\
\times & {\left.\left[\frac{\left(T-T_{j}\right)}{\left(T-T_{0}\right)} \frac{\alpha}{k(T)}-1\right]\right\}_{T=T_{\max }} . }
\end{aligned}
$$

For all $T_{j}<T<T_{\max }$, two distinct and positive real roots are obtained for $c_{A}$. The solid line in Fig. 8 illustrates the equilibrium line generated by eq. (51) for the given process parameters, in the feasible region of operation. The upper branch of the equilibrium line corresponds to low conversion/low residence time steady states, whereas the lower branch to high conversion/high residence time ones. Furthermore, a linear stability analysis can show that all the points of this equilibrium line are locally exponentially stable equilibrium points.

Analysis of the process zero dynamics

Setting $x_{1}=c_{A}, x_{2}=T, u=F$ and $y=T$, the dynamic model of the process can be easily put in the standard state-space form, with

$$
\begin{aligned}
f(x)= & {\left[\begin{array}{c}
-k\left(x_{2}\right) x_{1} \\
\gamma k\left(x_{2}\right) x_{1}-\alpha\left(x_{2}-T_{j}\right)
\end{array}\right], } \\
g(x)= & {\left[\begin{array}{c}
\frac{\left(c_{A 0}-x_{1}\right)}{V} \\
\frac{\left(T_{0}-x_{2}\right)}{V}
\end{array}\right], \quad h(x)=x_{2} . }
\end{aligned}
$$

The relative order of the output $y$ with respect to the manipulated input $u$ is then found to be $r=1$, since $L_{g} h(x)=\left[\left(T_{0}-x_{2}\right) / V\right] \neq 0$. Consider now the change of coordinates

$$
\zeta=\left[\begin{array}{c}
t(x) \\
h(x)
\end{array}\right]=\left[\begin{array}{c}
\frac{c_{A 0}-x_{1}}{T_{0}-x_{2}} \\
x_{2}
\end{array}\right]
$$

under which the original state-space model is transformed into its Byrnes-Isidori normal form

$$
\begin{aligned}
\dot{\zeta}_{1} & =F_{1}\left(\zeta_{1}, \zeta_{2}\right) \\
\dot{\zeta}_{2} & =F_{2}\left(\zeta_{1}, \zeta_{2}\right)+G_{2}\left(\zeta_{1}, \zeta_{2}\right) u \\
y & =\zeta_{2}
\end{aligned}
$$

where

$$
\begin{aligned}
F_{1}\left(\zeta_{1}, \zeta_{2}\right)= & -\gamma k\left(\zeta_{2}\right) \zeta_{1}^{2} \\
& +\left[\frac{\gamma k\left(\zeta_{2}\right) c_{A 0}}{\left(T_{0}-\zeta_{2}\right)}-\alpha \frac{\left(\zeta_{2}-T_{j}\right)}{\left(T_{0}-\zeta_{2}\right)}-k\left(\zeta_{2}\right)\right] \zeta_{1} \\
& +\frac{k\left(\zeta_{2}\right) c_{A 0}}{\left(T_{0}-\zeta_{2}\right)}
\end{aligned}
$$

The zero dynamics of the process is then defined as the dynamic system

$$
\dot{z}=F_{1}(z, y) \text {. }
$$

The stability of the zero dynamics locally around an equilibrium point can be easily determined using Liapunov's first theorem, i.e. based on the sign of

$$
\frac{\partial F_{1}}{\partial z}=\frac{\gamma k(y) c_{A 0}}{\left(T_{0}-y\right)}-\alpha \frac{\left(y-T_{j}\right)}{\left(T_{0}-y\right)}-k(y)-2 \gamma k(y) z .
$$

Transforming into the original coordinates, one obtains the following characterization of the state space:

Minimum-phase region:

$$
c_{A}>\frac{1}{2}\left\{c_{A 0}+\frac{\left(T-T_{0}\right)}{\gamma}\left[\frac{\left(T-T_{0}\right)}{\left(T-T_{0}\right)} \frac{\alpha}{k(T)}-1\right]\right\} .
$$


Nonminimum-phase region:

$$
c_{A}<\frac{1}{2}\left\{c_{A 0}+\frac{\left(T-T_{0}\right)}{\gamma}\left[\frac{\left(T-T_{j}\right)}{\left(T-T_{0}\right)} \frac{\alpha}{k(T)}-1\right]\right\} .
$$

The dividing line in a $c_{A}$ vs $T$ diagram is generated by the equation:

$$
c_{A}=\frac{1}{2}\left\{c_{A 0}+\frac{\left(T-T_{0}\right)}{\gamma}\left[\frac{\left(T-T_{j}\right)}{\left(T-T_{0}\right)} \frac{\alpha}{k(T)}-1\right]\right\}
$$

and is shown by the dashed line in Fig. 5 . It is interesting to observe that the point of the equilibrium line corresponding to the transition between the minimum-phase region and the nonminimum-phase region is exactly the point of maximum temperature (or double root in $c_{A}$ ).

\section{The control problem}

The desired operating equilibrium point for the process under consideration will be chosen at some location of the lower branch of Fig. 5, to achieve a compromise between maximizing conversion and maximizing production rate (this is typically determined taking into account process economics considerations). Clearly, in this region the process is nonminimum-phase, which makes the control problem especially challenging.

The initial operating point is assumed to he at the steady state: $x_{1 \mathrm{~s}}=4.29 \mathrm{kmol} \mathrm{m}^{-3}$, and $x_{2 s}=332 \mathrm{~K}$. Figure 6 illustrates the open-loop response of the process for a negative step change in $F$ from $0.203 \mathrm{~m}^{3} \mathrm{~h}^{-1}$ to $0.02 \mathrm{~m}^{3} \mathrm{~h}^{-1}$. One can observe the initial inverse response due to the unstable zero dynamics. Figure 10 also illustrates the open-loop response of the reactor, starting from a different steady state: $x_{1 s}=3.08$ $\mathrm{kmol} \mathrm{m}^{-3}$, and $x_{2 s}=312 \mathrm{~K}$, and for a positive step change in $F$ from $0.046 \mathrm{~m}^{3} \mathrm{~h}^{-1}$ to $0.20 \mathrm{~m}^{3} \mathrm{~h}^{-1}$. The nonlinear nature of the process is clearly documented in the significant differences of the response characteristics.

Following Wright and Kravaris, 1992 [see also Kravaris and Daoutidis (1990)] the ISE-optimal stati-

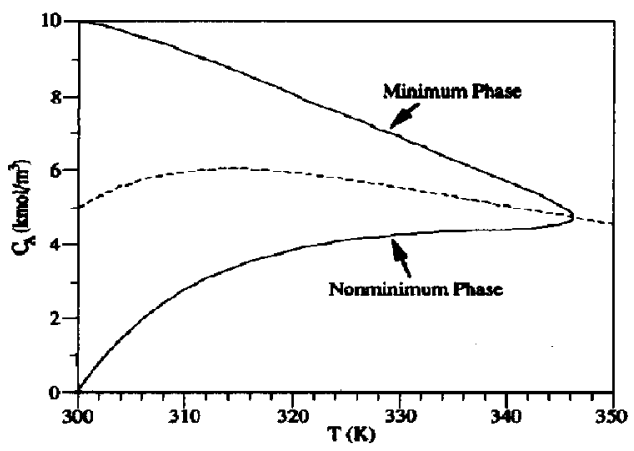

Fig. 5. Equilibrium line and dividing line for the CSTR.

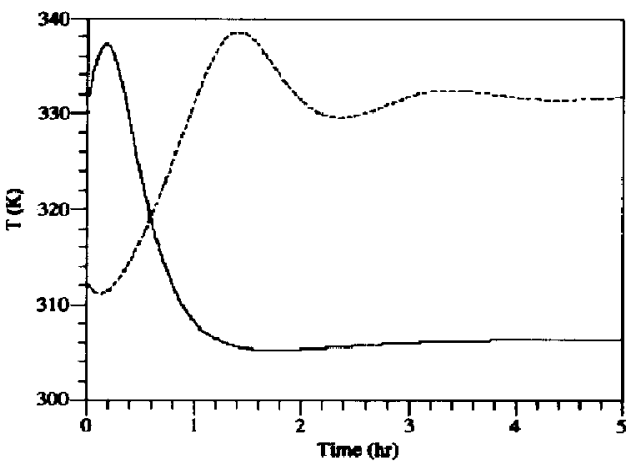

Fig. 6. Open-loop response of reactor temperature.

cally equivalent output map for this process can be found from the equation:

$$
h^{*}\left(\zeta_{1}, \zeta_{2}\right)=\zeta_{2}-\frac{2 F_{1}\left(\zeta_{1}, \zeta_{2}\right)}{\frac{\partial F_{1}}{\partial \zeta_{2}}\left(\zeta_{1}, \zeta_{2}\right)}
$$

where $F_{1}\left(\zeta_{1}, \zeta_{2}\right)$ is given in eq. (58). Clearly, the points in state space for which

$$
\frac{\partial F_{1}}{\partial \zeta_{2}}\left(\zeta_{1}, \zeta_{2}\right)=0
$$

represent a singular line for which $h^{*}$ is not welldefined. The output map of eq. (64) can be easily transformed in the original coordinates and used for the synthesis of the dynamic output feedback controller. More specifically, based on remark 7 and for $r=1$, the controller

$$
\begin{aligned}
\dot{w} & =f(w)+g(w) \frac{e+h(w)-h^{*}(w)-\gamma_{1} L_{f} h^{*}(w)}{\gamma_{1} L_{g} h^{*}(w)} \\
u & =\frac{e+h(w)-h^{*}(w)-\gamma_{1} L_{f} h^{*}(w)}{\gamma_{1} L_{g} h^{*}(w)}
\end{aligned}
$$

will induce the input/output dynamics:

$$
\gamma_{1} \frac{\mathrm{d}\left[h^{*}(x)\right]}{\mathrm{d} t}+h^{*}(x)=y_{s p}
$$

with $y=y_{s p}$ at steady state.

The adjustable parameter is chosen as $\gamma_{1}=0.25$ in all simulation runs that follow. Figure 7 illustrates the singular lines in the state space, for which $h^{*}(x)$ is not well-defined and the control law is not well-defined $\left[L_{g} h^{*}(x)=0\right]$.

In the first run, the reactor is initially assumed to be at the steady state: $x_{1 \mathrm{~s}}=4.29 \mathrm{kmol} \mathrm{m}^{-3}$, and $x_{2 s}=332 \mathrm{~K}$. A negative step change is imposed on the output set point, from $332 \mathrm{~K}$ to $322 \mathrm{~K}$. Figure 8 illustrates the profiles for the outputs $y^{*}$ and $y$, and the manipulated input $F$, under the controller of eq. (65). One can clearly observe the first-order response in the equivalent output $y^{*}$ according to the theoretical prediction, as well as the matching of $y$ and $y^{*}$ at steady 


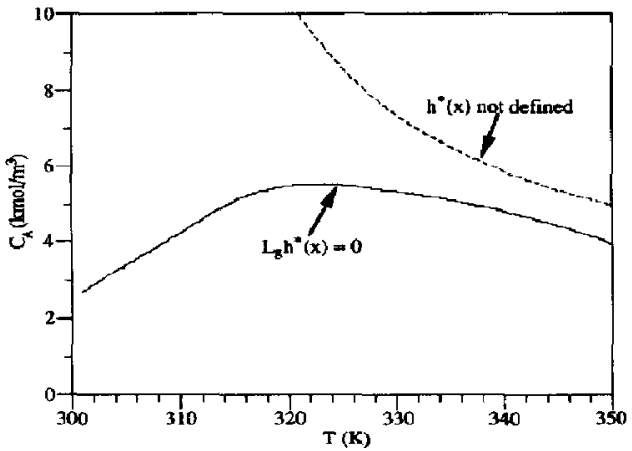

Fig. 7. Singular lines in the concentration/temperature diagram.

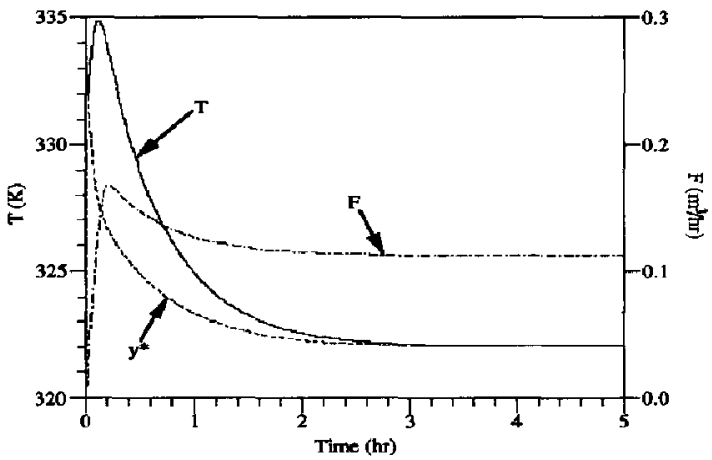

Fig. 8. Output and manipulated input profiles for set point change.

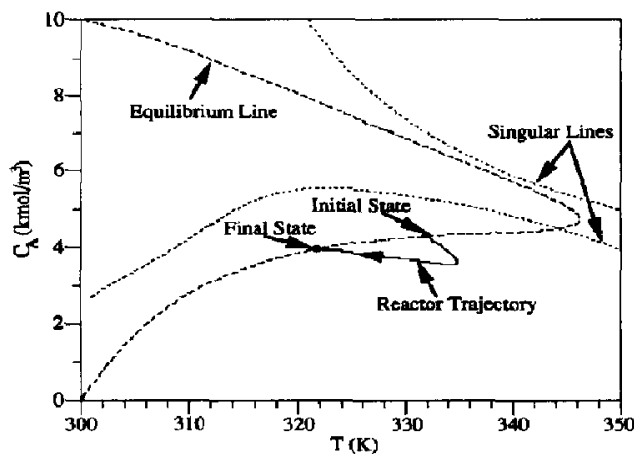

Fig. 9. Reactor trajectory in the concentration/temperature diagram for set point change.

state. Figure 9 illustrates the reactor trajectory which clearly remains away from the singular lines of the state space.

In the next two simulation runs, the same change in the set point is imposed, and the effect of initialization and modeling error on the controller performance is addressed. Figure 10 illustrates the profiles for the

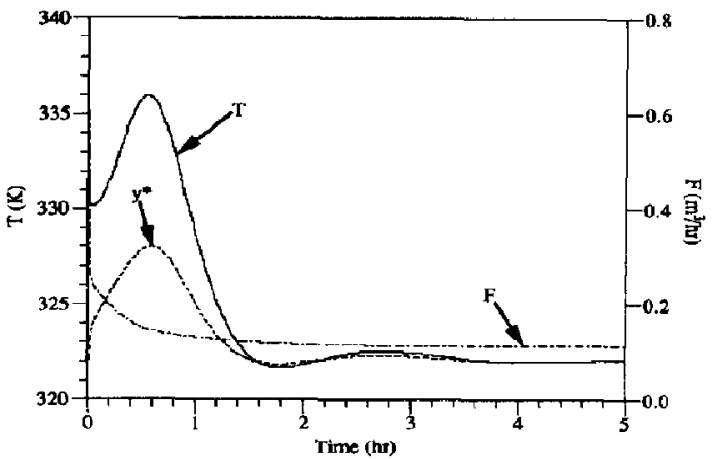

Fig. 10. Output and manipulated input profiles for set point change: effect of initialization error.

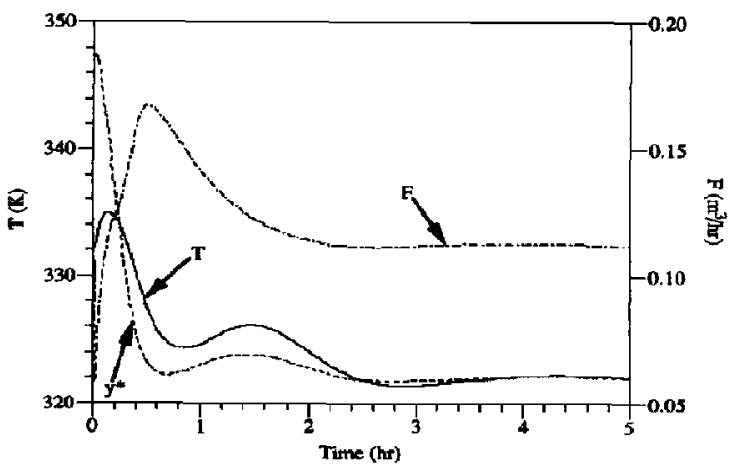

Fig. 11. Output and manipulated input profiles for set point change: effect of modeling error.

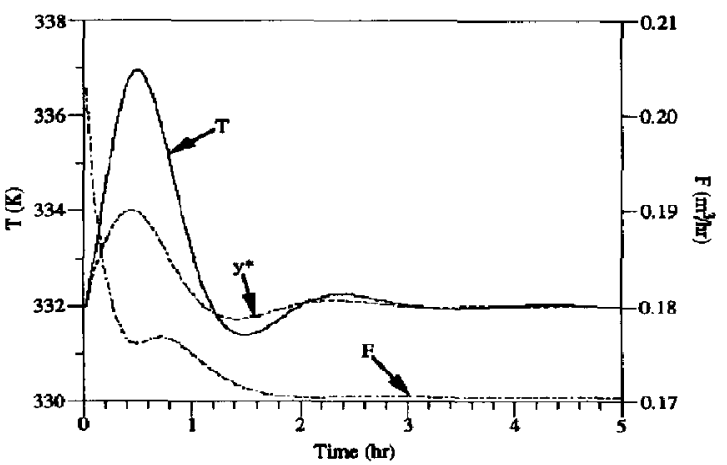

Fig. 12. Output and manipulated input profiles for rejection of inket temperature disturbance.

outputs $y^{*}$ and $y$, and the manipulated input $F$, for $w_{1}(0)=0.8 x_{1}(0)$ and $w_{2}(0)=x_{2}(0)+10$. Clearly, the controller performs very satisfactorily, despite the initialization error. Figure 11 illustrates the analogous profiles asuming an error of $10 \%$ in the model parameters $\gamma$ and $\alpha$. The controller is able to cope with the 


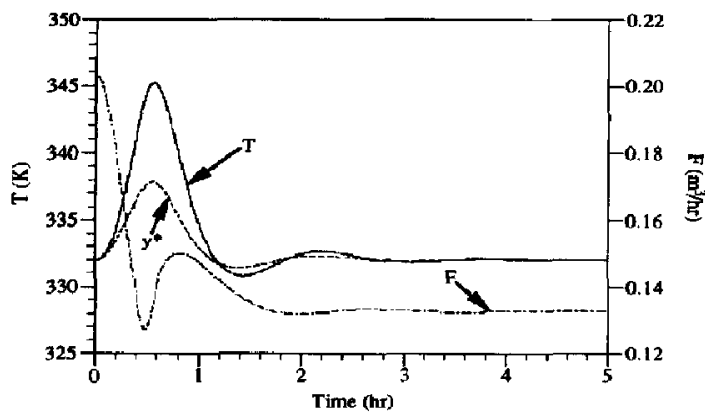

Fig. 13. Output and manipulated input profiles for rejection of inlet concentration disturbance.

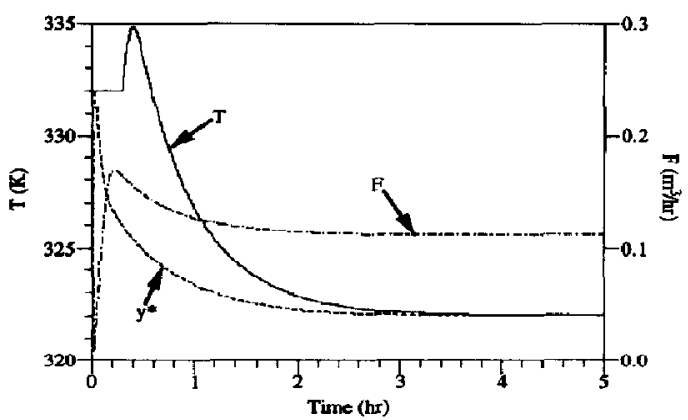

Fig. 14. Output and manipulated input profiles for set point change in the presence of dead-time (with no error in deadtime).

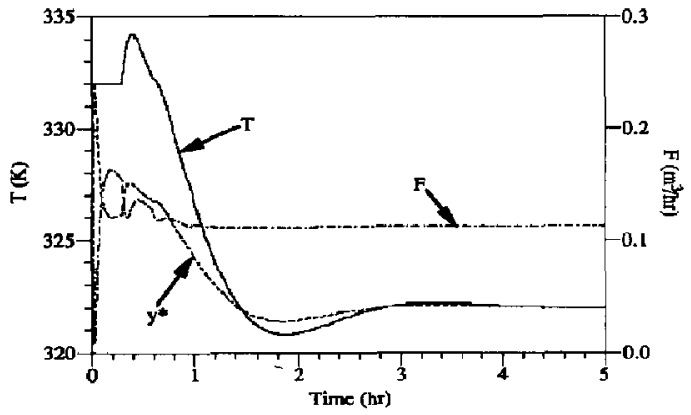

Fig. 15. Output and manipulated input profiles for set point change in the presence of dead-time (with error in deadtime).

modeling error and control the reactor to the new set point.

The next simulation runs address the disturbance rejection capability of the control scheme. The reactor is assumed to be at its nominal steady state $\left(x_{1 s}=4.29 \mathrm{kmol} \mathrm{m}^{-3}\right.$. and $\left.x_{2 s}=332 \mathrm{~K}\right)$. Initially, an unmeasured step increase of $5 \mathrm{~K}$ is assumed in the inlet temperature $T_{0}$. As can be seen in Fig. 12, the controller brings the system smoothly back to the set point. Then, an unmeasured step increase by $15 \%$ is assumed in the inlet concentration $c_{A 0}$, and the con-

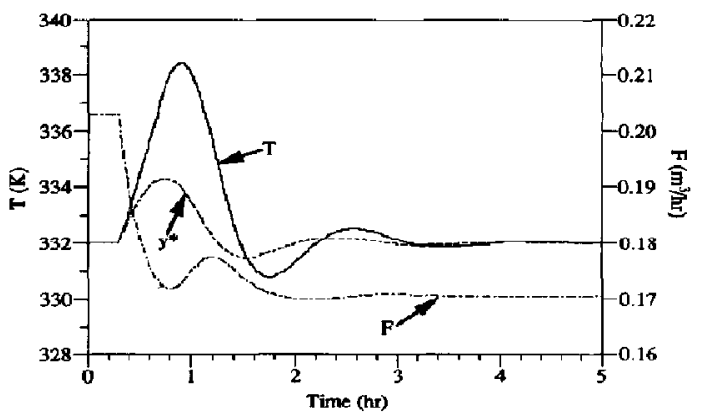

Fig. 16. Output and manipulated input profiles for rejection of inlet temperature disturbance in the presence of deadtime (with no error in deadtime).

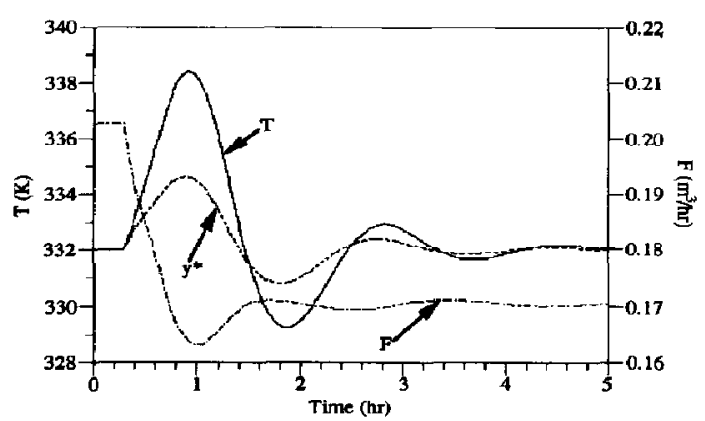

Fig. 17. Output and manipulated input profiles for rejection of inlet temperature disturbance in the presence of deadtime (with error in deadtime).

troller again brings the system successfully back to its set point (Fig. 13).

The final set of simulation runs addresses the case where, in addition to unstable zero dynamics, a deadtime $\theta=1000 \mathrm{~s}$ is present in the output map of the process; the controller of eq. (26) is used in all the simulations. For the same set point change as previously (from 332 to $322 \mathrm{~K}$ ), Fig. 14 shows the profiles for the outputs $y^{*}$ and $y$, and the manipulated input $F$, using the actual process deadtime in the controller equations. As predicted by the theory, a first-order response can be observed for $y^{*}$, while $y$ matches $y^{*}$ at steady state, after the initial delay in the response. Figure 15 shows the analogous profiles using $\theta=0 \mathrm{~s}$ in the controller equations. Even in the presence of such a substantial error in the deadtime, the controller performs very satisfactorily. Figure 16 shows the input and ouput profiles for the same unmeasured disturbance in the inlet temperature as previously (step increase of $5 \mathrm{~K}$ ), using the actual process deadtime in the controller equations. The controller successfully brings the system back to the set point. Figure 17 shows the analogous profiles using $\theta=0 \mathrm{~s}$ in the controller, and again illustrates the very satisfactory disturbance rejection capabilities of the controller, even in the presence of error in the deadtime. 


\section{CONCLUSIONS}

A general framework for the control of open-loop stable nonminimum-phase nonlinear processes was developed. A Smith-type abstract operator structured was proposed, providing a transparent stability analysis framework and allowing the unification of existing minimum- and nonminimum-phase compensation structures. A general nonminimum-phase compensation problem was posed and solved in a state-space framework. Reduced-order cuntroller realizations were derived, inducing a desired input/ output behavior for processes with unstable inverse dynamics and/or deadtime. A model state feedback structure was identified in the resulting controllers, and its key properties were studied in a general context. The developed control method was applied to a temperature control problem in an exothermic chemical reactor operating with cold feed. Extensive simulations were performed, demonstrating the excellent tracking and regulatory capabilities of the controller, and its robustness with respect to initialization errors, modeling errors and errors in deadtime.

Acknowledgement-Financial support from the National Science Foundation, Grant No. CTS-8912836 is gratefully acknowledged. The authors also wish to thank Masoud Soroush for his suggestions on the simulation example.

\section{NOTATION}

ca

$c_{A 0}$

C concentration of species $A$ in the reactor, $\mathbf{k m o l ~ m}^{-3}$

inlet concentration of species $A, \mathrm{kmol} \mathrm{m}^{-3}$

classical controller operator

controller operator in Smith-type operator structure

vector field

inlet flowrate, $\mathrm{m}^{3} \mathrm{~h}^{-1}$

vector field

output scalar field

auxiliary output scalar field

identity operator

reaction rate constant, $h^{-1}$

process operator

auxiliary process operator in Smith-type operator structure

controller operator

relative order of process output

relative order of auxiliary output

closed-loop operator

auxiliary closed-loop operator

time

reactor temperature, $K$

inlet temperature, $\mathbf{K}$

cooling jacket temperature, $\mathbf{K}$

manipulated input variable

volume of the reactor, $\mathrm{m}^{3}$

vector of controller state variables

vector of process state variables

output variable

output set point

auxiliary output

$\begin{array}{ll}\text { Greek letters } \\ \gamma_{k} & \text { adjustable parameters } \\ \zeta & \text { state vector in normal form coordinates } \\ \theta & \text { process deadtime } \\ \xi & \text { controller state variables } \\ \psi & \text { controller state variables }\end{array}$

\section{Mathematical symbols \\ $\epsilon$ belongs to \\ $\exists$ there exists}

$L_{f} h$ Lie derivative of the scalar field $h$ with respect to the vector field $f$

$L_{f}^{k} h \quad k$ th order Lie derivative of scalar field $h$ with respect to the vector field $f$

$\mathbb{R}$ real line

$\mathbb{R}^{n} \quad n$-dimensional Euclidean space

\section{REFERENCES}

Byrnes, C. I. and Isidori, A., 1985, Global feedback stabilization of nonlinear systems. Proceedings of the 24th IEEE $C D C, F t$. Lauderdale, FL, p. 1031.

Coulibajy, E. S., Maiti, S. and Brosilow, C., 1992, Internal model predictive control (IMPC), Paper No. 123j, 1992, AIChE Annual Meeting, Miami Beach, FL.

Daoutidis, P. and Alhumaizi, K., 1993, Feedforward/output feedback control of nonlinear processes. Proceedings 1993 American Control Conference, San Fransisco, CA, p. 1485.

Daoutidis, P. and Kravaris, C, 1992, Dynamic output feedback control of minimum-phase nonlinear processes. Chem. Engng Sci. 47, 837.

Desoer, C. A. and Vidyasagar, M., 1975, Feedback Systems: Input-Output Properties. Academic Press, New York.

Desoer, C. A. and Lin, C. A., 1984, Nonlinear unity-feedback systems and $Q$-parametrization. Int. $J$. Control $40,37$.

Desoer, C. A. and Kabuli, M. G., 1988, Stabilization and robustness of nonlinear unity-feedback system: factorization approach. Int. J. Control 47, 1133.

Hammer., J., 1984, On nonlinear systems, additive feedback and rationality. Int. J. Control 40, 953.

Kravaris, C. and Daoutidis, P., 1990, Nonlinear state feedback control of second-order nonminimum-phase monlinear systems. Comput. Chem. Engng 14, 439.

Kravaris, C. and Daoutidis, P., 1992, Output feedback controller realizations for open-loop stable nonlinear processes. Proceedings 1992 American Control Conference, Chicago, IL, p. 2576.

Kravaris, C. and Wright, R. A., 1989, Deadtime compensation for nonlinear processes. A.I.Ch.E. J. 35, 1535.

Isidori, A., 1989, Nonlinear Control Systems: An Introduction. Springer, Berlin, Heidelberg.

Juba, M. R. and Hamer, J. W., 1986, Progress and challenges in batch process control in: (Edited by $M$. Morari and T. J. McAvoy), Chemical Process Control-CPC III, p. 139. Elsevier, Amsterdam.

MeLellan, P. J., Harris, T. J. and Bacon, D. W., 1990, Error trajectory descriptions of nonlinear controller designs. Chem. Engng Sci. 45, 3017.

Misawa, E. A and Hedrick, J. K., 1989, Nonlinear observers-a state-of-the-art survey. J. Dyn. Systems, Measurement Control 111, 344.

Nijmeijer, H. and van der Schaft, A. J., 1990, Nonlinear Dynamical Control Systems. Springer, New York.

Ramanathan, S., Curl, R. L. and Kravaris, C., 1989, Dynamics and control of quasirational systems. A.I.Ch.E. J. 35, 1017.

Safonov, M. G., 1980, Stability and Robustness of Multivariable Feedback Systems. MIT Press, Cambridge, MA.

Soroush, M. and Kravaris, C., 1992a, Discrete-time nonlin- 
ear controller synthesis by input/output linearization. A.I.Ch.E. J. 38, 1923.

Soroush, M. and Kravaris, C., 1992b, A continuous-time formulation of nonlinear model predicitive control. Proceedings 1992 American Control Conference, Chicago, IL, p. 1561 .
Wright, R. A., 1990, Equivalent output formulations of nonlinear control problems. Ph.D. thesis., The University of Michigan, Ann Arbor.

Wright, R. A. and Kravaris, C., 1992, Nonminimum-phase compensation for nonlinear processes. A.I.Ch.E. J. 38, 26. 